\section{Average Fisher information maximisation in presence of cost-constrained measurements}

\section{B. Dulek and S. Gezici}

An optimal estimation framework is considered in the presence of costconstrained measurements. The aim is to maximise the average Fisher information under a constraint on the total cost of measurement devices. An optimisation problem is formulated to calculate the optimal costs of measurement devices that maximise the average Fisher information for arbitrary observation and measurement statistics. In addition, a closed-form expression is obtained in the case of Gaussian observations and measurement noise. Numerical examples are presented to explain the results.

Introduction: In estimation problems, the Cramer-Rao lower bound (CRLB) provides a lower bound on mean-squared errors (MSEs) of unbiased estimators. In addition, when the prior distribution of the unknown parameter is known, the Bayesian CRLB (BCRLB) can be calculated to obtain a lower bound on the MSE of any estimator [1]. The CRLB and BCRLB are quite useful in the analysis of estimation problems since (a) they provide lower bounds that can (asymptotically) be achieved by certain estimators (e.g. the maximum likelihood estimator), (b) they are easier to calculate than the MSE as their formulations do not depend on any specific estimator structure. Recently, a novel measurement device model has been proposed, and the problem of designing the optimal linear estimator is studied under a total cost constraint on the measurement devices [2]. Unlike previous studies, it is considered that each observation is measured by a measurement device, the accuracy of which depends on the cost spent on that device. In that way, a total cost constraint is taken into account and the optimal linear estimator design is performed under that constraint.

In this Letter, we consider the problem of minimising the BCRLB (equivalently, maximising the average Fisher information) at the outputs of measurement devices under the total cost constraint introduced in [2]. In other words, we propose a generic formulation for determining the optimal cost allocation among measurement devices in order to maximise the average Fisher information. We also obtain a closedform expression for the Gaussian case, and present numerical examples.

Optimal solution: Consider a scenario as in Fig. 1 in which a $K$-dimensional observation vector $\mathbf{x}$ is measured by $K$ measurement devices, and then the measured values in vector $\mathbf{y}$ are processed to estimate the value of parameter $\theta$. The measurement devices are modelled to introduce additive measurement noise denoted by $\mathbf{m}$. In other words, the probability density function (PDF) of $\mathbf{x}$ is indexed by parameter $\theta$, and the aim is to estimate that parameter based on the outputs of the measurement devices. Although a linear system model and a different problem formulation are considered in [2], motivations for that study can also be invoked for the system model in Fig. 1. It should be emphasised that the model in Fig. 1 presents a generic estimation framework in which measurements are processed by an estimator in order to determine the value of an unknown parameter. For example, in a wireless sensor network application, measurement devices correspond to sensors, which are used to estimate a parameter in the system, such as the temperature.

$\stackrel{\mathbf{x}}{\longrightarrow} \begin{gathered}\text { measurement } \\ \text { devices }\end{gathered} \stackrel{\mathbf{y}=\mathbf{x}+\mathbf{m}}{\longrightarrow} \stackrel{\text { estimator }}{\longrightarrow}$

Fig. 1 Observation vector $\boldsymbol{x}$ measured by $K$ measurement devices, and measurements $\boldsymbol{x}+\boldsymbol{m}$ are used by estimator to estimate value of unknown parameter $\theta$

To consider practical system constraints, we assume that there is a total cost constraint on the measurement devices, as proposed in [2]. Specifically, the total cost budget of the measurement devices cannot exceed $C$, which is specified by

$$
\frac{1}{2} \sum_{i=1}^{K} \log \left(1+\frac{\sigma_{x_{i}}^{2}}{\sigma_{m_{i}}^{2}}\right) \leq C
$$

where $\sigma_{x_{i}}^{2}$ denotes the variance of the $i$ th component of observation vector $\mathbf{x}$, and $\sigma_{m_{i}}^{2}$ is the variance of the $i$ th measurement device (i.e., the $i$ th component of $\mathbf{m}$ ). In other words, it is assumed that a measurement device has a higher cost if it can perform measurements with a lower measurement variance (i.e. with higher accuracy). Various motivations for the cost constraint in (1) can be found in [2].

To maximise the estimation accuracy, we consider the maximisation of average Fisher information, or equivalently the minimisation of the BCRLB at the output of the measurement devices. The main motivation for the suggested approach is that an optimal cost assignment strategy can be obtained by solving such an optimisation problem without assuming a specific estimator structure. In addition, it is known that some estimators, such as the maximum a-posteriori probability estimator, can (asymptotically) achieve the BCRLB; hence, the minimisation of the BCRLB corresponds to the (approximate) minimisation of the MSE for certain estimators [1]

For an arbitrary estimator $\widehat{\theta}$, the BCRLB on the MSE is expressed as

$$
\operatorname{MSE}\{\widehat{\theta}\}=\mathrm{E}\left\{(\widehat{\theta}(\mathbf{y})-\theta)^{2}\right\} \geq\left(\mathrm{J}_{\mathrm{D}}+\mathrm{J}_{\mathrm{P}}\right)^{-1}
$$

where $J_{D}$ and $J_{P}$ denote the information obtained from observations and prior knowledge, respectively, which are stated as

$$
\mathrm{J}_{\mathrm{D}}=\mathrm{E}\left\{\left(\frac{\partial \log p_{\mathbf{Y}}^{\theta}(\mathbf{y})}{\partial \theta}\right)^{2}\right\}, \quad \mathrm{J}_{\mathrm{P}}=\mathrm{E}\left\{\left(\frac{\partial \log w(\theta)}{\partial \theta}\right)^{2}\right\}
$$

with $p_{\mathbf{Y}}^{\theta}(\mathbf{y})$ and $w(\theta)$ representing the PDF of $\mathbf{Y}$ and the prior PDF of the parameter, respectively. As $\mathrm{J}_{\mathrm{P}}$ depends only on the prior PDF, it is independent of the cost of the measurement devices. Therefore, the aim is to maximise $\mathrm{J}_{\mathrm{D}}$, which is defined as the average Fisher information, under the cost constraint in (1). To specify this optimisation problem, it is assumed that the observation is independent of the measurement noise; hence, $p_{\mathbf{Y}}^{\theta}(\mathbf{y})$ in (3) can be expressed more explicitly as the convolution of the PDFs of $\mathbf{x}$ and $\mathbf{m}$; i.e. $p_{\mathbf{Y}}^{\theta}(\mathbf{y})=\int p_{\mathbf{X}}^{\theta}(\mathbf{y}-\mathbf{m}) p_{\mathbf{M}}(\mathbf{m}) d \mathbf{m}$. In addition, it is reasonable to assume that each measurement device introduces independent noise, in which case $p_{\mathbf{M}}(\mathbf{m})$ becomes $p_{\mathbf{M}}(\mathbf{m})=p_{M_{1}}\left(m_{1}\right) \ldots p_{M_{K}}\left(m_{K}\right)$. As discussed in [2], the cost of a measurement device can be expressed as a function of its measurement noise variance (see (1)). Each measurement noise component can be modelled as $m_{i}=\sigma_{m_{i}} \tilde{m}_{i}$, where $\tilde{m}_{i}$ denotes a zero-mean unit-variance random variable with a known PDF $p_{\tilde{M}_{i}}$, and $\sigma_{m_{i}}^{2}$ represents the variance of the measurement device, which determines its cost as defined in (1). Hence, the PDF of the $i$ th measurement noise can be expressed as $p_{M_{i}}(m)=\sigma_{m_{i}}^{-1} p_{\tilde{M}_{i}}\left(\sigma_{m_{i}}^{-1} m\right)$.

Based on (1) and (3), the optimal cost assignment problem can be formulated as

$$
\begin{aligned}
& \max _{\left\{\sigma_{m_{i}}^{2}\right\}_{i=1}^{K}} \int w(\theta) \int_{\mathbf{R}^{K}} \frac{1}{p_{\mathbf{Y}}^{\theta}(\mathbf{y})}\left(\frac{d p_{\mathbf{Y}}^{\theta}(\mathbf{y})}{d \theta}\right)^{2} d \mathbf{y} \\
& \text { subject to } \frac{1}{2} \sum_{i=1}^{K} \log \left(1+\frac{\sigma_{x_{i}}^{2}}{\sigma_{m_{i}}^{2}}\right) \leq C
\end{aligned}
$$

It is noted that the expectation operator for the calculation of $\mathrm{J}_{\mathrm{D}}$ in (3) is over both $\theta$ and $\mathbf{Y}$, resulting in the objective function in (4). From the discussions in the previous paragraph, we have $p_{\mathbf{Y}}^{\theta}(\mathbf{y})=$ $\int p_{\mathbf{X}}^{\theta}(\mathbf{y}-\mathbf{m}) \prod_{i=1}^{K} \sigma_{m_{i}}^{-1} p_{\tilde{M}_{i}}\left(\sigma_{m_{i}}^{-1} m_{i}\right) d \mathbf{m}$, which becomes

$$
\begin{aligned}
p_{\mathbf{Y}}^{\theta}(\mathbf{y})= & \prod_{i=1}^{K} \sigma_{m_{i}}^{-1} \int_{-\infty}^{\infty} p_{X_{i}}^{\theta}\left(y_{i}-m_{i}\right) p_{\tilde{M}_{i}}\left(\sigma_{m_{i}}^{-1} m_{i}\right) d m_{i} \\
& =\prod_{i=1}^{K} \int_{-\infty}^{\infty} p_{X_{i}}^{\theta}\left(y_{i}-\sigma_{m_{i}} m\right) p_{\tilde{M}_{i}}(m) d m
\end{aligned}
$$

in the case of independent observations. In fact, the objective function in (4) can be written as the sum of $K$ components in that case (see (3)) as

$$
\sum_{i=1}^{K} \int w(\theta) \int_{-\infty}^{\infty} \frac{1}{p_{Y_{i}}^{\theta}(y)}\left(\frac{d p_{Y_{i}}^{\theta}(y)}{d \theta}\right)^{2} d y
$$

where

$$
p_{Y_{i}}^{\theta}(y)=\int_{-\infty}^{\infty} p_{X_{i}}^{\theta}\left(y-\sigma_{m_{i}} m\right) p_{\tilde{M}_{i}}(m) d m
$$

Since the optimisation problem in (4) provides a generic formulation that is valid for any observation PDF, the problem can be non-concave in 
general. Hence, global optimisation tools such as particle swarm optimisation and differential evolution can be used to obtain the solution [3].

Special case: In the case of independent Gaussian observations and measurement noise, it is possible to obtain closed-form solutions of the optimisation problem in (4). To that aim, let the observation vector $\mathbf{x}$ have independent Gaussian components denoted as $X_{i} \sim N\left(\theta, \sigma_{x_{i}}^{2}\right)$ for $i=1, \ldots, K$, and let each measurement noise component have independent zero-mean Gaussian distribution with variance $\sigma_{m_{i}}^{2}$. In that case, the average Fisher information $\mathrm{J}_{\mathrm{D}}$ can be calculated as $\sum_{i=1}^{K}\left(\sigma_{m_{i}}^{2}+\sigma_{x_{i}}^{2}\right)^{-1}$. Hence, the aim becomes the maximisation of $\sum_{i=1}^{K}\left(\sigma_{m_{i}}^{2}+\sigma_{x_{i}}^{2}\right)^{-1}$ over $\sigma_{m_{1}}^{2}, \ldots, \sigma_{m_{K}}^{2}$ under the constraint in (1). It is noted that both the objective function and the constraint are convex in this optimisation problem. Since the maximum of convex functions over convex sets has to occur at the boundary [4], the cost constraint becomes equality, and the solution of the optimisation problem can be obtained by using Lagrange multipliers [4], resulting in the following algorithm for the optimal cost allocation:

$$
\sigma_{m_{i}}^{2}=\left\{\begin{array}{cl}
\frac{\sigma_{x_{i}}^{4}}{\gamma-\sigma_{x_{i}}^{2}}, & \text { if } \sigma_{x_{i}}^{2}<\gamma \\
\infty & \text { if } \sigma_{x_{i}}^{2} \geq \gamma
\end{array} \text { with } \gamma=\left(2^{2 C} \prod_{i \in S_{K}} \sigma_{x_{i}}^{2}\right)^{1 /\left|S_{K}\right|}\right.
$$

where $S_{K}=\left\{i \in\{1, \ldots, K\}: \sigma_{m_{i}}^{2} \neq \infty\right\}$ and $\left|S_{K}\right|$ denotes the number of elements in set $S_{K}$. In other words, if the observation noise variance is larger than a threshold $\gamma$, a measurement device with infinite variance (that is, with zero cost) is considered; namely, that observation is not measured at all. On the other hand, for observations with variances smaller than $\gamma$, the noise variance of the corresponding measurement device is determined according to the formulation in (6), which assigns low measurement variances (high costs) to observations with low variances.

Table 1: Measurement variances and corresponding Fisher information for optimal strategy (see (6)), strategy 1, strategy 2

\begin{tabular}{|c|c|c|c|c|c|}
\hline & $\sigma_{m_{1}}^{2}$ & $\sigma_{m_{2}}^{2}$ & $\sigma_{m_{3}}^{2}$ & $\sigma_{m_{4}}^{2}$ & Fisher information \\
\hline Optimal & 0.0097 & 0.3973 & 3.533 & $\infty$ & 10.45 \\
\hline Strategy 1 & 0.4373 & 0.4373 & 0.4373 & 0.4373 & 4.252 \\
\hline Strategy 2 & 0.0032 & $\infty$ & $\infty$ & $\infty$ & 9.688 \\
\hline
\end{tabular}
1.3

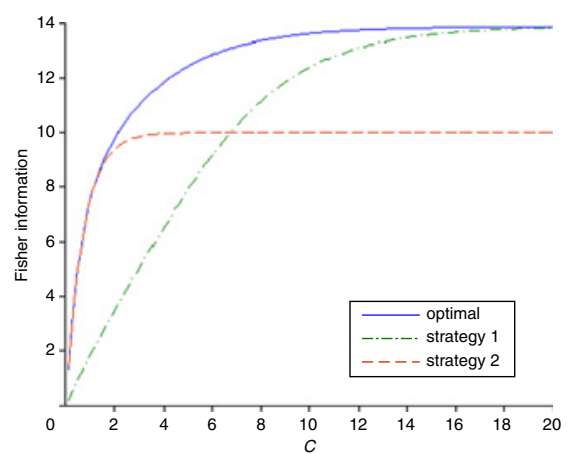

Fig. 2 Fisher information against total cost C for optimal strategy, strategy 1 , strategy 2 where $\sigma_{x_{1}}{ }^{2}=0.1, \sigma_{x_{2}}{ }^{2}=0.5, \sigma_{x_{3}}{ }^{2}=0.9$, and ${\sigma_{x_{4}}}^{2}=1.3$ optimal

strategy 1

strategy 2
Alternative strategies: Instead of the optimal cost assignment strategy specified in (4), one can also consider the following simple alternatives. Strategy 1 (equal measurement device variances): In this strategy, it is assumed that measurement devices with equal variances are used for all observations; i.e. $\sigma_{m_{i}}^{2}=\sigma_{m}^{2}, i=1, \ldots, K$. Then, the cost constraint in (4) can be used with equality, and $\sigma_{m}^{2}$ is simply obtained as the smallest positive real root of $\prod_{i=1}^{K}\left(1+\sigma_{x_{i}}^{2} / \sigma_{m}^{2}\right)=2^{2 C}$. If the observation variances are also equal, $\sigma_{m}^{2}$ becomes $\sigma_{m}^{2}=\sigma_{x}^{2} /\left(2^{2 C / K}-1\right)$.

Strategy 2 (all cost to the best observation): In this case, the total budget $C$ is spent on the best observation, which has the smallest variance. If the $b$ th observation is the best one, the cost constraint in (4) can be used to calculate the variance of the measurement noise for that observation as $\sigma_{m_{b}}^{2}=\sigma_{x_{b}}^{2} /\left(2^{2 C}-1\right)$. For all the other observations, the corresponding measurement variances are set to infinity (i.e. no measurements are taken from those observations).

Results and conclusions: To provide numerical examples of the results in the preceding Sections, consider a scenario with independent Gaussian observations and measurement noise. Let $C=2.5$, $\sigma_{x_{1}}^{2}=0.1, \sigma_{x_{2}}^{2}=0.5, \sigma_{x_{3}}^{2}=0.9$, and $\sigma_{x_{4}}^{2}=1.3$. In Table 1 , the variances of the measurement devices and the corresponding Fisher information values are shown for the proposed optimal strategy (see (6)), strategy 1 and strategy 2 . It is observed that the optimal strategy assigns smaller variances (larger costs) to observations with smaller variances, and achieves the maximum Fisher information as expected. For further investigations, Fig. 2 illustrates the Fisher information versus the total budget $C$ for different strategies. It is observed that the Fisher information in strategy 2 , which assigns all the cost to the best observation, converges to $1 / \sigma_{x_{1}}^{2}$ as expected (since $\sigma_{m_{1}}^{2}$ converges to zero as $C$ increases). On the other hand, strategy 2 and the optimal strategy converge for very small values of $C$ since the optimal strategy involves assigning all the cost to the best observation if $C$ is small. Regarding strategy 1 , it converges to the optimal strategy for large $C$, and significant deviations are observed for intermediate values of $C$. Overall, the optimal cost assignment strategy yields the highest Fisher information in all the cases, and indicates the opportunity to achieve high estimation accuracy.

(C) The Institution of Engineering and Technology 2011

11 March 2011

doi: $10.1049 / \mathrm{el} .2011 .0686$

One or more of the Figures in this Letter are available in colour online.

B. Dulek and S. Gezici (Department of Electrical and Electronics Engineering, Bilkent University, Bilkent, Ankara 06800, Turkey)

E-mail: gezici@ee.bilkent.edu.tr

\section{References}

1 Van Trees, H.L.: 'Detection, estimation, and modulation theory, Part I' (Wiley-Interscience, New York, 2001)

2 Ozcelikkale, A., Ozaktas, H.M., and Arikan, E.: 'Signal recovery with cost-constrained measurements', IEEE Trans. Signal Process., 2010, 58, (7), pp. 3607-3617

3 Storn, R., and Price, K.: 'Differential evolution - a simple and efficient heuristic for global optimisation over continuous spaces', J. Global Optimiz., 1997, 11, pp. 341-359

4 Rockafellar, R.: 'Convex analysis' (Princeton University Press, 1972) 